\title{
Acute Macular Neuroretinopathy Related to Alcoholic Hepatitis
}

\author{
Grace Anne Mc Cabe ${ }^{a}$ William Gordon Campbella, b \\ Thomas Gordon Campbell ${ }^{a, b, c}$ \\ aDepartment of Ophthalmology, Royal Melbourne Hospital, Parkville, VIC, Australia; \\ ${ }^{b}$ Department of Ophthalmology, Royal Victorian Eye and Ear Hospital, East Melbourne, VIC, \\ Australia; 'Centre for Eye Research Australia, East Melbourne, VIC, Australia; ${ }^{\mathrm{d}}$ Department of \\ Surgery, University of Melbourne, Melbourne, VIC, Australia
}

\author{
Keywords \\ Retinopathy $\cdot$ OCT angiography $\cdot$ Retinal blood flow
}

\begin{abstract}
A 33-year-old woman admitted for acute alcoholic hepatitis was referred to the ophthalmology department with an acute onset paracentral scotoma of the left eye. On examination, best-corrected visual acuity was Snellen 6/4 in the right eye and 6/9 in the left eye. Dilated left fundus examination revealed wedge-shaped changes at the macula. Spectral-domain ocular coherence tomography (SD-OCT) initially revealed a small cuff of subfoveal fluid and band-like hyperreflectivity extending outwards from the outer plexiform layer consistent with acute macular neuroretinopathy (AMN). Four days later, repeat SD-OCT was performed and it demonstrated resolution of the subfoveal fluid and disruption of the outer retinal layers. At the 6-week follow-up, the patient had no improvement in her symptoms and OCT angiography demonstrated coarsening and microvascular changes in both the deep vascular plexus and the choriocapillaris. To our knowledge, this is the first case of AMN in association with acute hepatitis. Although the exact pathophysiology of AMN remains obscure, this case highlights the benefits of multimodal retinal imaging and aims to bring attention to the possible association of AMN with alcoholic hepatitis.
\end{abstract}

(C) 2021 The Author(s).

Published by S. Karger AG, Basel

\section{Introduction}

Acute macular neuroretinopathy (AMN) is a rare retinal disorder first described by Bos and Deutman [1], characterized by acute onset photopsias, paracentral scotomas, and mild visual impairment. Macular lesions have a characteristic reddish-brown appearance, with the apices pointing towards the fovea often in a petaloid, tear drop, or wedge-shaped configu- 
Mc Cabe et al.: Acute Macular Neuroretinopathy Related to Alcoholic Hepatitis with Multimodal Imaging

ration [1]. The disease predominantly affects females, most commonly in the third decade of life, and symptoms may or may not resolve [2]. The most frequently reported associations are a non-specific flu-like illness, use of oral contraceptives (OCPs), and exposure to either adrenaline or ephedrine. The pathophysiology and anatomical correlates of AMN are a matter of some controversy but either choroidal or deep retinal ischaemia are regarded as the most likely aetiology [3]. We describe a case of AMN associated with an episode of acute alcoholic hepatitis in a young female.

\section{Case Presentation}

A 33-year-old female of African heritage was admitted with abdominal pain, vomiting, and anorexia, on a background of known use alcohol disorder. Blood work was suggestive of acute alcoholic hepatitis with an AST:ALT ratio >2:1: AST $533 \mathrm{U} / \mathrm{L}$, ALT $192 \mathrm{U} / \mathrm{L}$, and bilirubin 30 U/L. Haemoglobin, white cell count, and platelet count were within normal limits. Testing for hepatitis A, B, and C was negative. A magnetic resonance cholangiopancreatography conducted to evaluate for gallstones did not demonstrate intra-biliary pathology. Abdominal ultrasound demonstrated severe fatty liver, in addition to acute on chronic pancreatitis. In addition, the lipase was elevated to $126 \mathrm{U} / \mathrm{L}$, further supporting this diagnosis.

Her condition was managed conservatively with fluids, vitamin repletion, anti-emetics, and analgesia. On day 4 of admission, she was referred to the ophthalmology unit with acute onset of a scotoma in her left eye. She had no other relevant past medical or ocular history and denied the use of OCPs, other medications, or illicit substances. In addition, she denied fever, night sweats, or other viral symptoms and reported no recent travel.

On examination, best-corrected visual acuity was 20/13.33 in the right eye and 20/30 in the left eye. Intraocular pressure was $14 \mathrm{~mm} \mathrm{Hg}$ bilaterally. Dilated left fundus examination revealed wedge-shaped changes at the macula, as well as 3 cotton wool spots on the superior arcade and an adjacent blot haemorrhage. The right eye was normal. Left fundus photography and infrared reflectance demonstrated subtle linear brown lesions in a pseudo-macular star appearance (Fig. 1). Computerized perimetry demonstrated a paracentral scotoma (Fig. 2), with a corresponding Amsler grid illustration by the patient (Fig. 2c).

Fig. 1. a, b Colour fundal photo and infrared reflectance images of the left posterior poles at presentation demonstrating a hyporeflective wedge-shaped lesion at the left macula with linear hyperreflective bands superiorly and nasally. Infrared reflectance image of the left posterior poles at day 4, demonstrating a hyporeflective wedge-shaped lesion at the left macula (c). Multicolour image of left posterior pole at day 45 (d).
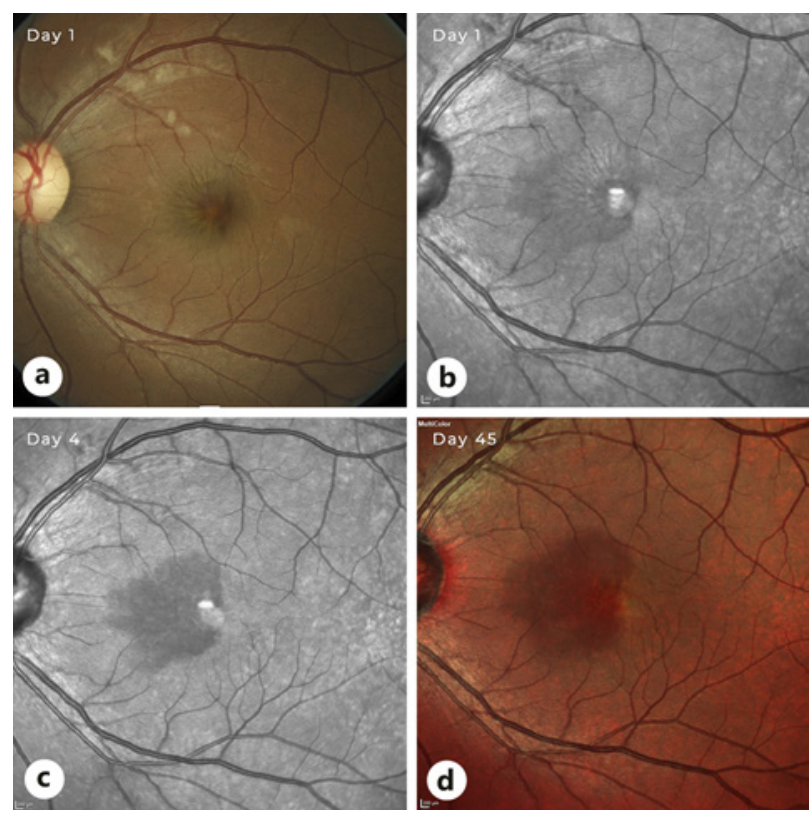


\section{Case Reports in Ophthalmology}

\begin{tabular}{|c|c|}
\hline \multicolumn{2}{|c|}{ Case Rep Ophthalmol 2021;12:293-298 } \\
\hline DOI: $10.1159 / 000513186$ & $\begin{array}{l}\text { (c) } 2021 \text { The Author(s). Published by S. Karger AG, Basel } \\
\text { www.karger.com/cop }\end{array}$ \\
\hline
\end{tabular}

Mc Cabe et al.: Acute Macular Neuroretinopathy Related to Alcoholic Hepatitis with Multimodal Imaging
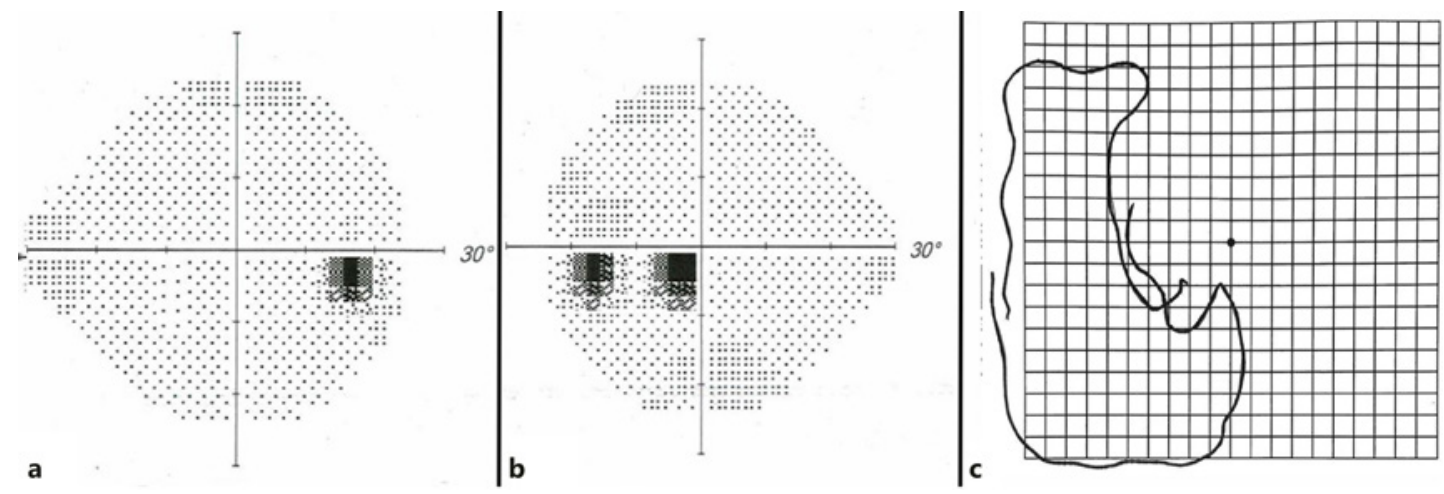

Fig. 2. a, b Automated perimetry of the right and left eyes demonstrating a left central scotoma. c Amsler grid drawn by the patient to demonstrate scotoma from left eye.

Spectral-domain ocular coherence tomography (SD-OCT) (Spectralis; Heidelberg Engineering, Heidelberg, Germany) initially revealed a small cuff of subfoveal fluid and band-like hyperreflectivity extending outwards from the outer plexiform layer consistent with AMN (Fig. 3a). Four days later, repeat SD-OCT was performed. It showed resolution of the subfoveal fluid and disruption of the outer retinal layers (Fig. 3b). The patient denied any improvement in visual symptoms and described a persistent paracentral scotoma. Infrared reflectance imaging showed classic wedge-shaped lesions extending outwards from the fovea (Fig. 1c). Fluorescein angiography was unremarkable with no evidence of macular ischaemia.

Six weeks following the onset of her symptoms, the patient was re-admitted, this time with acute pancreatitis. At this time, she described a persistent paracentral scotoma without improvement. Repeat SD-OCT revealed marked outer retinal thinning in the affected area, with disruption of the inner segment/outer segment zone (Fig. 3c). OCT angiography (Spec-

Fig. 3. a Ocular coherence tomography of the left macula during the acute phase, demonstrating a hyperreflective band extending outwards from the outer plexiform layer consistent with an AMN lesion. b Left macula at day 4. Note the disruption of the outer retinal layers in the area corresponding to the acute macular neuroretinopathy lesion. c Left macula at day 45 . Note the loss of outer retinal layers. AMN, acute macular neuroretinopathy.
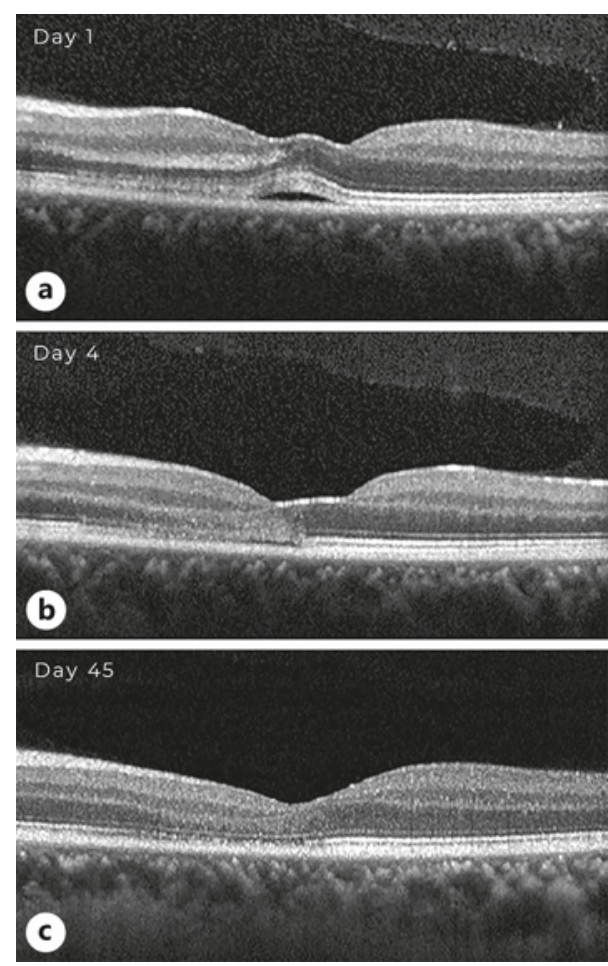
Mc Cabe et al.: Acute Macular Neuroretinopathy Related to Alcoholic Hepatitis with Multimodal Imaging

tralis), now available at this follow-up, demonstrated preservation of the superficial and intermediate vascular plexi but coarsening and microvascular changes in both the deep vascular plexus and the choriocapillaris (Fig. 4).

\section{Discussion}

AMN is a rare retinal pathology of uncertain incidence; a recent systematic review found 101 cases between 1975 and 2014 [2]. It has been reported to occur both unilaterally or bilaterally, and it appears to have a propensity for young healthy women of reproductive age. An initial case series in 1975 reported an association with OCPs [1]; since this time, flu-like illness or fever, exposure to adrenaline or ephedrine, trauma, systemic shock, thrombocytopaenia, anaemia, hyperviscosity, and dehydration have all been linked to AMN [2, 4]. A recurring theme amongst these associated factors has been the potential for transient, systemic vasculopathy to incite AMN.

It is of interest that in addition to the findings of AMN in this patient, there were also features of inner retinal ischaemia including cotton wool spots. This association has been reported previously [5] and highlights that this condition results from a systemic vasculopathy. As AMN becomes increasingly recognized it is likely that the spectrum of ophthalmic associations will be broadened.

Multimodal imaging, including SD-OCT, has improved our ability to recognize the lesions of AMN and its characteristic deep retinal changes, leading to increased recognition of this disorder. The hyperreflective band in the outer retinal layers in the acute phase is diagnostic of the condition and is thought to reflect ischaemia of the outer plexiform (OPL) and outer nuclear layers. Although it is well accepted that AMN reflects an ischaemic process, it is a matter of some controversy as to whether the retinal or choroidal vascular bed is involved [2, $3,6-8]$. This uncertainty may arise in part from the fact that the OPL is thought to be the watershed zone between the retinal and choroidal circulations. The relevance of this watershed zone is highlighted by the clinically distinct paracentral acute middle maculopathy described by Sarraf et al. [9], which is characterized by hyperreflective bands extending from the OPL inwards. A cogent explanation of the distinction between these 2 entities is that AMN

Fig. 4. a, b Optical coherence tomography angiography of the deep capillary plexus of the right and left fovea at day 45. There is coarsening of the deep capillary plexus in the area corresponding to the AMN lesion. c, d Optical coherence tomography angiography of the choriocapillaris of the right and left fovea at day 45 . There are marked changes of the choriocapillaris underlying the lesion. AMN, acute macular neuroretinopathy.
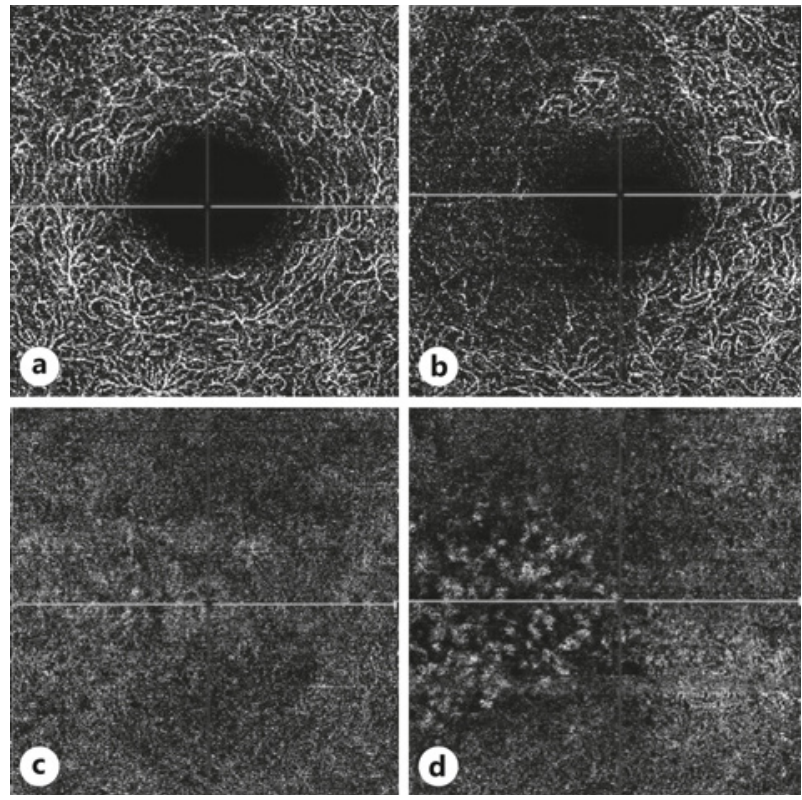
represents focal choriocapillaris dysfunction whereas paracentral acute middle maculopathy represents an ischaemic process within the deep vascular complex of the retina [10]. Interestingly however OCT angiography has failed to definitively delineate the involved vasculature; some reports highlight involvement of the deep vascular complex of the retina [2], whereas others implicate the choriocapillaris [7]. Interestingly, Casalino and colleagues have demonstrated OCT-A changes at both the level of the choriocapillaris and the deep capillary plexus [8]. It is unclear whether these lesions, especially those in the deep capillary plexus, are a cause or a consequence of the original insult.

Three distinct pathophysiological explanations could account for the diversity of OCT-A findings in AMN. Firstly, it is possible that there are inter-individual differences in the relative contributions of the retinal and choroidal circulations to the watershed zone of the OPL, and therefore in some individuals' ischaemic insult to the deep capillary plexus causes AMN whereas in others this area is entirely perfused by the choriocapillaris, and thus AMN reflects choriocapillaris dysfunction. Secondly, it is possible that the deep capillary plexus changes are a consequence of the original insult. In this interpretation, ischaemic insult to the outer retinal layers leads to a downstream decrease in cellular activity either via transynaptic degeneration or decreased synaptic activity, resulting in pruning of the vascular bed. OCT-A did not become available at our unit until $>1$ month after our patient's initial presentation, so this theory could explain her follow up OCT-A findings. Thirdly, it is possible that the ischaemia-induced retinal changes result in artefacts in the OCT-A, either through shadowing from the hyperreflective ischaemic retina, segmentation errors due to retinal thinning or thickening, or low flow-related resolution errors.

To our knowledge, alcoholic hepatitis is a new association of AMN. While the exact pathophysiology of AMN remains somewhat elusive, this case highlights the benefits of multimodal retinal imaging and aims to bring attention to the possible association of AMN with alcoholic hepatitis.

\section{Acknowledgements}

We would like to acknowledge the Department of Medical Photography at the Royal Melbourne Hospital. TGC is supported by the Hector Maclean Scholarship.

\section{Statement of Ethics}

The authors obtained informed written consent from the patient for publication of this report, including consent to publish images. The research was conducted ethically in accordance with the Declaration of Helsinki. The paper is exempt from Ethics Committee approval as per Melbourne Health HREC guidelines on case reports.

\section{Conflict of Interest Statement}

The authors have no conflicts of interest to declare.

\section{Funding Sources}

Thomas Gordon Campbell was supported by the Hector McClean scholarship.

\section{Karger'}


Mc Cabe et al.: Acute Macular Neuroretinopathy Related to Alcoholic Hepatitis with Multimodal Imaging

\section{Author Contributions}

Grace Mc Cabe: contribution to patient care, acquisition of data, conception of the work, and drafting the manuscript. William Campbell: revising critically for important intellectual content, reviewing and editing of the manuscript. Thomas Campbell: contribution to patient care, analysis and interpretation of data, and drafting the work. All authors read and approved the final manuscript.

\section{References}

1 Bos PJ, Deutman AF. Acute macular neuroretinopathy. Am J Ophthalmol. 1975;80(4):573-84.

2 Bhavsar KV, Lin S, Rahimy E, Joseph A, Freund KB, Sarraf D, et al. Acute macular neuroretinopathy: a comprehensive review of the literature. Surv Ophthalmol. 2016;61(5):538-65.

3 Lee SY, Cheng JL, Gehrs KM, Folk JC, Sohn EH, Russell SR, et al. Choroidal features of acute macular neuroretinopathy via optical coherence tomography angiography and correlation with serial multimodal imaging. JAMA Ophthalmol. 2017;135(11):1177.

4 Munk MR, Jampol LM, Cunha Souza E, De Andrade GC, Esmaili DD, Sarraf D, et al. New associations of classic acute macular neuroretinopathy. Br J Ophthalmol. 2015;100(3):389-94.

5 Kuriakose RK, Chin EK, Almeida DRP. An atypical presentation of acute macular neuroretinopathy after nonocular trauma. Case Rep Ophthalmol. 2019 Jan 10 [cited 2020 Nov 11];10(1):1-4. Available from: https:// www.karger.com/Article/FullText/496144.

6 Fawzi AA, Pappuru RR, Sarraf D, Le PP, McCannel CA, Sobrin L, et al. Acute macular neuroretinopathy: longterm insights revealed by multimodal imaging. Retina. 2012;32(8):1500-13.

7 Thanos A, Faia LJ, Yonekawa Y, Randhawa S. Optical coherence tomographic angiography in acute macular neuroretinopathy. JAMA Ophthalmol. 2016;134(11):1310-4.

8 Casalino G, Arrigo A, Romano F, Munk MR, Bandello F, Parodi MB. Acute macular neuroretinopathy: pathogenetic insights from optical coherence tomography angiography. Br J Ophthalmol. 2019;103(3):410-4.

9 Rahimy E, Kuehlewein L, Sadda SR, Sarraf D. Paracentral acute middle maculopathy: what we knew then and what we know now. Retina. 2015;35(10):1921-30.

10 Dansingani KK, Freund KB. Paracentral acute middle maculopathy, acute macular neuroretinopathy: related and distinct entities. Am J Ophthalmol. 2015;160:1-3. 